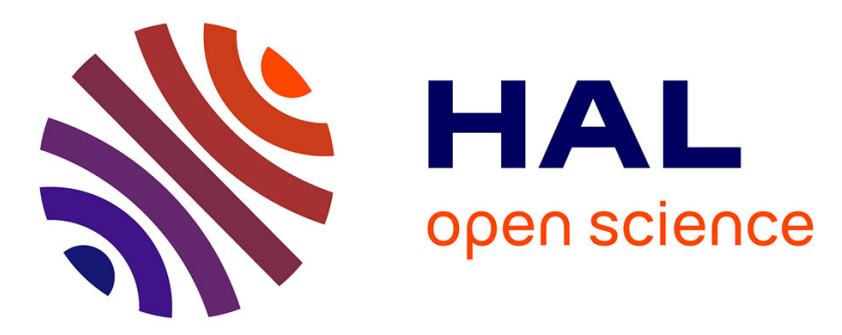

\title{
A generalization of flatness to nonlinear systems of partial differential equations. Application to the command of a flexible rod
}

Francois Ollivier, Alexandre Sedoglavic

\section{- To cite this version:}

Francois Ollivier, Alexandre Sedoglavic. A generalization of flatness to nonlinear systems of partial differential equations. Application to the command of a flexible rod. Nonlinear Control Systems 2001, Jul 2001, St Petersbourg Russie. inria-00126994

\section{HAL Id: inria-00126994 \\ https://hal.inria.fr/inria-00126994}

Submitted on 27 Jan 2007

HAL is a multi-disciplinary open access archive for the deposit and dissemination of scientific research documents, whether they are published or not. The documents may come from teaching and research institutions in France or abroad, or from public or private research centers.
L'archive ouverte pluridisciplinaire HAL, est destinée au dépôt et à la diffusion de documents scientifiques de niveau recherche, publiés ou non, émanant des établissements d'enseignement et de recherche français ou étrangers, des laboratoires publics ou privés. 


\title{
A GENERALIZATION OF FLATNESS TO NONLINEAR SYSTEMS OF PARTIAL DIFFERENTIAL EQUATIONS. APPLICATION TO THE COMMAND OF A FLEXIBLE ROD
}

\author{
Published In Proceedings of the 5th IFAC Symposium \\ "Nonlinear Control Systems" (Saint Petersburg, Russia, \\ July 4-6 2001), A. Kurzhanski and A. Fradkov, Eds., \\ vol. 1, Elsevier, pp. 196-200.
}

\author{
François Ollivier* and Alexandre Sedoglavic *,1 \\ * Laboratoire GAGE \\ UMS CNRS-École polytechnique $n^{\circ} 658$ \\ F-91128 Palaiseau, France \\ \{ollivier,sedoglavic\}@gage.polytechnique.fr
}

\begin{abstract}
We introduce a concept of differential flatness for systems described by nonlinear partial differential equations. It generalizes the now classical notion of differential flatness for finite differential systems and its recent extensions to linear partial differential equations. We apply it to the motion planning of a very flexible rod, outside of the linear approximation range.
\end{abstract}

Keywords: Systems governed by nonlinear partial differential equations, differential flatness, finite difference methods, motion planning.

\section{INTRODUCTION}

This work is based on the concept of differential flatness for finite dimensional systems introduced in (Fliess et al., 1995), which has already been extended to infinite dimensional linear system (see (Fliess and Mounier, 1999), (Laroche et al., 2000) and (Lynch and Rudolph, 2000)). We propose here a generalization of this concept to systems described by nonlinear partial differential equations. It is in some way a "brute force" generalization: we use finite difference methods to replace PDE by a finite system of ODE, and use then the classical definition of differential flatness.

However, we cannot consider as flat a system of non-linear PDE which would only be the limit of a sequence of flat discretizations. We also need that the sequence of flat outputs converges and that the sequence of solutions parametrized by arbitrary functions in a convenient functional space converges too. So, our standpoint can no more rely, at this step, on pure algebra. We also need some analytical considerations.

1 With a support of the France-Argentina program ECOS-SECyT, action n ${ }^{\circ}$ A99E06.
Hereafter, the term infinite dimensional system denotes a system defined by partial differential equations; we do not consider delay system and refer to (Fliess and Mounier, 1998) and (Mounier, 1995) for this topic. We refer to (Yanenko, 1971) for a link with numerical analysis, and to (Lions, 1968) or (Bensoussan et al., 1992 \& 1993) for a more general study of systems governed by PDE.

We illustrate this generalized notion of flatness in a restricted situation: the control of a flexible rod. A one dimensional system of this kind may be discretized as a chain of small segments, the control acting at one extremity. Such finite dimensional systems are known for being flat. Even in this case, theoretical investigations of analytical nature may be tedious. We restrict here to experiments by computer computations. We are able to obtain a suitable control with a good approximation with a discretization with no more than 20 elements.

\section{FLATNESS OF FINITE DIMENSIONAL SYSTEM}

The concept of differential flatness was introduced in (Fliess et al., 1995) for non-linear finite di- 
mensional systems. A model is described by a differential system

$$
\dot{x}_{i}=f_{i}(x, u), \quad i=1, \ldots, n,
$$

where the $x_{i}$ denote the state variables and $u=\left(u_{1}, \ldots, u_{m}\right)$ the control vector.

A system is said to be flat if there exist some flat outputs $y=h\left(x, u, \ldots, u^{(j)}\right)$ with $j \in \mathbb{N}$ such that the components of $y$ and all their derivatives are functionally independent and such that we can parametrize every solution $(x, u)$ of $(1)$ in some dense open set by means of the flat output $y$ and its derivatives up to a finite order $i$ :

$$
\begin{aligned}
& x=F\left(y, \ldots, y^{(i)}\right), \\
& u=G\left(y, \ldots, y^{(i)}\right) .
\end{aligned}
$$

There is no uniqueness of the flat output even if there is usually a favourite flat output expressing physical properties.

The concept of flatness can be seen as a nonlinear generalization of the Kalman's controllability and of the Brunovský decomposition. Hence, every linear controllable system is flat.

Example. In order to illustrate these definitions and to introduce some notions used in the sequel, let us consider the following family of linear systems indexed by an integer $n$ :

$$
H_{n}\left\{\begin{aligned}
\dot{\theta}_{1} & =\left(\theta_{2}-\theta_{1}\right) n^{2}, \\
\dot{\theta}_{2} & =\left(\theta_{3}-2 \theta_{2}+\theta_{1}\right) n^{2}, \\
& \vdots \\
\dot{\theta}_{n-1} & =\left(\theta_{n}-2 \theta_{n-1}+\theta_{n-2}\right) n^{2} .
\end{aligned}\right.
$$

If we consider $\theta_{n}$ as a command, and the remaining $\theta_{i}, i<n$ as state variables, then it is quite straightforward that $\theta_{1}$ is a flat output of the system $H_{n}$ for any integer $n$. Indeed, an easy computation shows that:

$$
\begin{aligned}
& \theta_{2}=\theta_{1}+\frac{1}{n^{2}} \dot{\theta}_{1}, \\
& \theta_{3}=\theta_{1}+\frac{3}{n^{2}} \dot{\theta}_{1}+\frac{1}{n^{4}} \ddot{\theta}_{1}, \\
& \theta_{4}=\theta_{1}+\frac{6}{n^{2}} \dot{\theta}_{1}+\frac{5}{n^{4}} \ddot{\theta}_{1}+\frac{1}{n^{6}} \theta_{1}^{(3)}, \text { etc. }
\end{aligned}
$$

More precisely, we have the following general relation:

$$
\theta_{i}=2 \theta_{i-1}-\theta_{i-2}+\frac{\dot{\theta}_{i-1}}{n^{2}}, \text { for } 2 \leq i \leq n,
$$

which leads to the following parametrizations:

$$
\theta_{i}=\sum_{j=0}^{i-1} \frac{\left(\begin{array}{c}
i+j-1 \\
2 j
\end{array}\right)}{n^{2 j}} \theta_{1}^{(j)} \text { for } 2 \leq i \leq n .
$$

Thus, by imposing a desired trajectory on the flat output $\theta_{1}$, we can obtain the control $\theta_{n}$ which generates this trajectory without any integration of differential equations. Furthermore, this control defines the associated trajectories of the other state variables $\theta_{i}$ for $2 \leq i \leq n-1$.

\section{FLATNESS AND DISCRETIZATION OF INFINITE DIMENSIONAL SYSTEMS}

The concept of flatness has been extended to the infinite dimensional linear case using the operational calculus of Mikusiński which gives a substitute for the Laplace transformation. We refer to (Fliess and Mounier, 1999) and the references therein for an introduction to this standpoint and for some examples.

We propose another approach based on the same general strategy: we associate to the original infinite dimensional system a sequence of finite dimensional ones and the problem is reduced to studying the flatness of these last systems.

The main interest of our proposition is that, contrarily to the approach based on the operational calculus of Mikusiński, it enables the control of some infinite dimensional nonlinear systems. Proceeding by steps, we will first introduce it on a linear example, and show our results are basically equivalent to our predecessors'.

\subsection{Boundary control problem of the heat equation}

Let us denote by $\theta(t, x)$ the temperature along a rod of length 1 and by $u(t)$, the control variable. We assume that we heat - or cool - the extremity of the rod at $x=1$ while the other end at $x=0$ is perfectly insulated. Thus, this system is modelled by:

$$
\begin{cases}\partial_{t} \theta-\partial_{x^{2}} \theta & =0 \\ \partial_{x} \theta(t, 0) & =0 \\ \theta(t, 1) & =u(t)\end{cases}
$$

In (Fliess and Mounier, 1999) and (Laroche et al., 2000), the authors give an explicit open loop control able to steer the one-dimensional heat equation with control on the boundary from any state to any other state. The flat output $y$ is the temperature of the insulated end $\theta(t, 0)$. Suppose that $y\left(t_{0}+t\right)$ admits a power series expansion at $t_{0}$ for all $0 \leq t_{0} \leq 1$,

$$
y\left(t_{0}+t\right)=\sum_{i=0}^{\infty} c_{i} \frac{t^{i}}{i !},
$$

Fliess et al. obtain the solution:

$$
\theta\left(t_{0}, x\right)=\sum_{i=0}^{\infty} \frac{c_{i}}{(2 i) !} x^{2 i}
$$

If we want to heat the rod in order to make it go from a uniform temperature $\tau_{1}$ to another uniform 
temperature $\tau_{2}$, we may use a smooth function such that its time derivatives of any order are equal to zero at $t=0$ and at $t=T$ and which is constant for $t \notin[0, T]$ :

$$
\phi(t)=\left\{\begin{array}{cl}
0 & \text { if } t<0, \\
\frac{\int_{0}^{t / T} \exp (-1 / t(1-t)) d t}{\int_{0}^{1} \exp (-1 / t(1-t)) d t} & \text { if } t \in[0, T], \\
1 & \text { if } t>T .
\end{array}\right.
$$

We take then $y(t)=\tau_{1}+\left(\tau_{2}-\tau_{1}\right) \phi(t)$. This function is such that $\left|y^{(i)}(t)\right|<i !^{2} / t^{(2 i)}, y$ is said to be a Gevrey function of order 2. A Gevrey function $f$ of order $\alpha$ is such that $\left|f^{(i)}(\cdot)\right|<M A^{i} i !^{\alpha}$.

It is proved in (Laroche et al., 2000) that the series (4) expressing $\theta_{0}(t, x)$ is convergent and defines a $\mathcal{C}^{\infty}$ function. It is Gevrey of order $\alpha$ in $t$ and order 1 in $x$ when $y$ is Gevrey of order $\alpha<2$. As all the derivatives of $y$ are 0 at $t=0$ and $t=1$, the temperature $\theta$ of the rod will be uniform at those two times.

Semidiscretization and flatness. We consider the classical finite difference method used in numerical integration of partial differential equations (see (Yanenko, 1971)). This method is based on Taylor expansion of $\theta$ with respect to the space and time variables.

Hereafter, the time is leaved unchanged and the space is discretized in order to approximate the original system by an ordinary differential system and to use its property of flatness. We use the following relation for this semidiscretization:

$\theta(t, x+h)-\theta(t, x)=h \partial_{x} \theta(t, x)+\frac{h^{2}}{2 !} \partial_{x^{2}} \theta(t, x)+\mathcal{O}\left(h^{3}\right)$.

Hence, the space variable is discretized with uniform space step $h=1 / n$. If we denote $\theta(t, i / n)$ by $\theta_{i}(t)$, for $1 \leq i \leq n-1$, this leads to the following well-known relations:

$$
\partial_{x^{2}} \theta_{i}=\frac{\theta_{i+1}-2 \theta_{i}+\theta_{i-1}}{h^{2}}+\mathcal{O}\left(h^{3}\right) .
$$

Thus, the solutions of the system (3) are $a p$ proximated at precision at least $\mathcal{O}\left(1 / n^{2}\right)$ by the solutions of the following system:

$$
\left\{\begin{array}{l}
\partial_{t} \theta_{n, i}=\left(\theta_{n, i+1}-2 \theta_{n, i}+\theta_{n, i-1}\right) n^{2}, 1 \leq i \leq n-1 \\
\theta_{n, 0}-\theta_{n, 1}=0 \\
\theta_{n, n}=u(t)
\end{array}\right.
$$

These semidiscretizations are similar to the ordinary differential systems $H_{n}$ presented in section 2. Hence, the original infinite dimensional system (3) can be approximated at an arbitrary precision by a flat finite dimensional system.

Asymptotic behavior. The flat output $y$ has an obvious physical meaning. It corresponds to the temperature of the insulated end, as in the previous approach.
In order to express the temperature at a point of the rod, we define the Ramanujan $Q$-distribution:

$$
Q(p, q)=\frac{p !}{(p-q) ! p^{q}} .
$$

Using it, we may parametrize the solutions of $H_{n}$, using $y=\theta_{n, 1}$ and its derivatives:

$$
\theta_{n, i}=\sum_{j=0}^{i-1} Q(i+j-1,2 j) \frac{y^{(j)}}{(2 j) !}\left(\frac{i+j-1}{n}\right)^{2 j}
$$

Considering a point $x \in[0,1]$, we can express from (2) the temperature at $x$ as the limit $\lim _{n \rightarrow \infty} \theta_{n,\lfloor n x\rfloor}$, if such a limit exists.

The summands of these expressions depend both on the index of summation and on the number $n$ of discretizations. Thus, we can determine a control $u(t)$ such that the temperature at the endpoint of the rod satisfies a given smooth temperature profile $y(t)$ for $t \geq 0$, provided that the sequence $y^{(i)}(t)$ has limited growth for all $x>0$.

As mentioned above, it is the case for Gevrey functions of order $\alpha<2$ and some Gevrey function of order 2 such that the series (4) is convergent. For such functions, the properties of the Ramanujan $Q$-distribution (see (Sedgewick and Flajolet, 1996)), imply that the $\operatorname{limit}_{n \rightarrow \infty} \lim _{n,\lfloor n x\rfloor}$ exists and that we have the relations:

$$
\begin{aligned}
\theta(t, x) & \left.=\lim _{n \rightarrow \infty} \theta_{n,\lfloor n x\rfloor}=\sum_{j \in \mathbb{N}} \frac{y^{(j)}(t)}{(2 j) !} x^{2 j}, \forall x \in\right] 0,1[, \\
u(t) & =\lim _{n \rightarrow \infty} \theta_{n}=\sum_{j \in \mathbb{N}} \frac{y^{(j)}(t)}{(2 j) !} .
\end{aligned}
$$

We recover in this way the conclusions of the previous cited works.

\subsection{Toward a definition}

The above approach relies on the following points:

- the infinite dimensional system is approximated by a sequence of flat finite dimensional systems;

- the discretizated systems converge to the original system;

- the sequence of flat outputs has a limit;

- the instantiation of the flat output can be chosen in a class of function such that the parametrizations exist, and rich enough to go from one equilibrium point to another.

We call a system described by a system of nonlinear partial differential equations with limit conditions flat if is satisfies those four conditions.

\section{MOTION PLANNING OF A HIGHLY FLEXIBLE ROD}

Hereafter, we illustrate the above method by performing some numerical simulations concerning 
the control of a infinite dimensional nonlinear system. In the last subsection, we will study the convergence of the sequence of solutions.

\subsection{A Lagrangian nonlinear model}

Let us consider a planar highly flexible rod of weight $m$ with length $L$. The origin of the $\operatorname{rod} s=0$ has a controlled position $\left(u_{1}(t), u_{2}(t)\right)$. At the other end, a mass $M$ is fixed. The configuration of the rod at time $t$ is described by the angle $\omega(t, s)$ of the tangent to the rod at distance $s$ of the origin. The orientation $\omega(t, 0)=u_{3}(t)$ of the rod at $s=0$ is also controlled.

Hence, the position of the point at distance $s$ is $(x(t, s), y(t, s))$, where:

$$
\begin{aligned}
& x(t, s)=x(t, 0)+\int_{0}^{s} \cos (\omega(t, \sigma)) d \sigma, \\
& y(t, s)=y(t, 0)+\int_{0}^{s} \sin (\omega(t, \sigma)) d \sigma .
\end{aligned}
$$

In order to modelise the behavior of the rod, we use the classical variational description, using the Lagrangian expression:

$$
\int_{0}^{L} C\left(\partial_{s} \omega\right)^{2}-\rho\left(\left(\partial_{t} x\right)^{2}+\left(\partial_{t} y\right)^{2}\right)
$$

where $C$ is the flexibility coefficient and $\rho=m / L$.

We obtain then the following nonlinear EulerLagrange equation:

$$
\frac{C}{\rho} \partial_{s^{2}} \omega+\sin (\omega) \int_{s}^{L} \partial_{t^{2}} x-\cos (\omega) \int_{s}^{L} \partial_{t^{2}} y=0 .
$$

Semidiscretization. As explained in section 3.1, we consider the semidiscretization of these expressions. Hence, if we denote $x(t, i / n)($ resp. $y(t, i / n)$, $\omega(t, i / n))$ by $x_{n, i}(t)$ (resp. $\left.y_{n, i}(t), \omega_{n, i}(t)\right)$, this leads to:

$$
\begin{aligned}
& x_{n, i}=x_{n, n}+\frac{L}{n} \sum_{j=i}^{n-1} \cos \left(\omega_{n, j}\right), \\
& y_{n, i}=y_{n, n}+\frac{L}{n} \sum_{j=i}^{n-1} \sin \left(\omega_{n, j}\right) .
\end{aligned}
$$

We use a variant of the discretization (5), approximating $\partial_{x^{2}} \omega_{n, i}$ by

$$
\frac{\omega_{n, i}-2 \omega_{n, i-1}+\omega_{n, i-2}}{(L / n)^{2}}+\mathcal{O}\left(n^{-3}\right) .
$$

Using the semidiscretizations (7) and (8), the relation (6) can be discretized as follows:

$$
\begin{aligned}
\frac{C}{\rho} \Delta_{n, i} & =\cos \left(\omega_{n, i}\right)\left(M \ddot{y}_{n, n}+\frac{m}{n} \sum_{j=i}^{n} \ddot{y}_{n, j}\right) \\
& -\sin \left(\omega_{n, i}\right)\left(M \ddot{x}_{n, n}+\frac{m}{n} \sum_{j=i}^{n} \ddot{x}_{n, j}\right)
\end{aligned}
$$

for $0 \leq i \leq n-2$; thus, we have:

$$
\omega_{i-2}=2 \omega_{i-1}-\omega_{i}-\frac{C}{\rho}\left(\frac{L}{n}\right)^{2} \Delta_{n, i}
$$

Assuming that the mass at the end has inertial momentum $J$ leads to the following boundary condition $\omega_{s}=J \omega_{t t}$ with discretizations:

$$
\omega_{n, n-1}=\omega_{n, n}+\frac{L}{n} \frac{J \ddot{\omega}_{n, n}}{C} .
$$

From formulas (9) and (10), we can express the values of all state variables $\omega_{n, i}$ for $0 \leq i \leq n$, knowing only the three functions $\omega_{n, n}, x_{n, n}$ and $y_{n, n}$ and their derivatives. So, all the discretized models are flat, the flat outputs being the position and the orientation of the mass $M$, viz. $x_{L}, y_{L}, \omega_{L}$.

\subsection{Numerical simulation results}

Desired behavior. The control objective will be to assign a circular motion of $\pi$ rad to the mass, as shown in figure 1. Furthermore, this motion is completed in $T \mathrm{~s}$ and the rod is straight and still at the begining and the end of the motion.

In order to guarantee the absence of vibrations at the end of the motion, we use the smooth function $\phi$ defined above, with $T=1$.

Flat output. The requested behavior of the rod is obtained by the following instantiation of $\omega_{L}$ and the resulting relations:

$$
\begin{aligned}
& \omega_{L}=\pi \cdot \phi(t), \\
& x_{L}=L \cos \left(\omega_{L}\right), \\
& y_{L}=L \sin \left(\omega_{L}\right) .
\end{aligned}
$$

The presented simulation corresponds to the

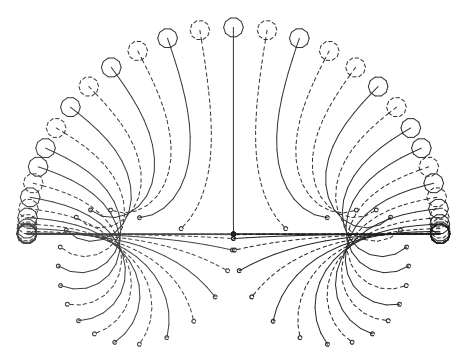

Fig. 1. Position of the rod through the time.

following values for the parameters of the rod:

$$
L=1 \mathrm{~m}, M=2 \mathrm{~kg}, m=0.5 \mathrm{~kg},
$$

$J=.009375 \mathrm{~kg} \mathrm{~m}^{2}, T=1 \mathrm{~s}, C=20 \mathrm{~N} \mathrm{~m}^{2}$. 
Figure 1 displays the motion of this rod through the time. The computations were done with a discretization of 15 mesh points and with a floating point precision of 10 digits; it requires a couple of minutes on a standard personal computer.

Experimental study of the asymptotic behavior. We conclude the present example by some numerical indications on the asymptotic behavior of the used approximations. Let us denote by $u_{n}(t)$ the approximation of the command $(x(t, 0), y(t, 0), \theta(t, 0))$ obtained with $n$ mesh points.

We sketch in figure 2 the variation $v_{i, j}=\left\|u_{i}-u_{j}\right\|$ between two approximated commands for increasing $i, j$. The presented curves correspond to the

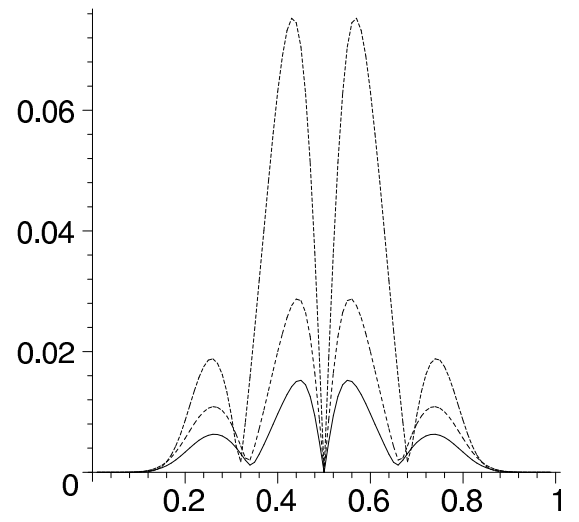

Fig. 2. experimental convergence of the command.

variations $v_{5,10}, v_{10,15}$ and $v_{15,20}$. We have

$$
\max _{t \in[0, T]} v_{5,10}>2 \max _{t \in[0, T]} v_{10,15}>4 \max _{t \in[0, T]} v_{15,20} .
$$

Further numerical computations indicate that the approximated command is likely convergent.

The above method leads to an open-loop control but it could be completed by a closed-loop stabilization of the trajectory error with respect to the desired motion (see (Fliess et al., 1995)).

\section{CONCLUSION}

We have proposed a method for the boundary control of some infinite dimensional nonlinear systems. This method is inspired by a generalization of the flatness notion to infinite dimensional systems. We have shown that in the linear case we recover the results of our predecessors who used Mikusiński's operational calculus and module theory.

In the nonlinear case, we were able to study the control of a nonlinear flexible rod, by numerical simulations. A careful study of the convergence of such a method remains to be performed. However the experimental fast convergence of the discretized models is encouraging. It should also be underlined that the computed command can very well be certified by numerical and practical experiments, so that a delay in the theoretical study is not a fatal trouble for applications.

In (Laroche et al., 2000), the use of divergent series is also considered, using "smallest term summation". Our approach could also be modified in order to include such a standpoint. The computations are fast enough for one dimensional bodies. We hope to be able in the future to consider the control of two dimensional objects.

\section{REFERENCES}

Bensoussan, A., G. Da Prato, M.C. Delfour and S.K. Mitter (1992 \& 1993). Representation and Control of Infinite Dimensional Systems. Vol. 1 \& 2. Birkhäuser.

Fliess, Michel and Hugues Mounier (1998). Controllability and observability of linear delay systems: an algebraic approach. ESAIM: control optimisation and calculus of variations 3, 301-314.

Fliess, Michel and Hugues Mounier (1999). Tracking control and $\pi$-freeness of infinite dimensional linear system. In: Dynamical systems, control coding, computer vision (G. Picci and D.S. Gilliam, Eds.). Birkhäuser. pp. 45-68.

Fliess, Michel, Jean Lévine, Philippe Martin and Pierre Rouchon (1995). Flatness and defect of nonlinear systems: introductory theory and examples. International journal of control 61(6), 1327-1361.

Laroche, Béatrice, Philippe Martin and Pierre Rouchon (2000). Motion planning for the heat equation. International journal of robust and nonlinear control 10(8), 629-643.

Lions, J.-L. (1968). Contrôle optimal des systèmes gouvernés par des équations aux dérivées partielles. Dunod.

Lynch, Alan F. and Joachim Rudolph (2000). Flatness-based boundary control of a nonlinear parabolic equation modelling a tubular reactor. In: Nonlinear control in the year 2000 (Alberto Isidori, F. Lamnabhi-Lagarrigue and W. Respondek, Eds.). Vol. 259 of Lecture notes in control and information sciences. Springer. London. pp. 45-54.

Mounier, Hugues (1995). Propriétés structurelles des systèmes linéaires à retards : aspects théoriques et pratiques. Thèse de doctorat. Université Paris-Sud. Orsay.

Sedgewick, Robert and Philippe Flajolet (1996). An Introduction to the Analysis of Algorithms. Addison-Wesley.

Yanenko, Nicolay Nicolayevich (1971). The method of fractional steps. Springer-Verlag. 